\title{
Classificação morfofuncional dos dentes de quati, Nasua nasua ${ }^{1}$
}

\author{
Naira C.G. Pieri², Celina A.F. Mançanares², Bruno Bertassoli², Jussara M.N. Lima ${ }^{2}$, \\ Juliana M.Thomaz ${ }^{2}$ e Ana F. Carvalho2*
}

\begin{abstract}
Pieri N.C.G., Mançanares C.A.F., Bertassoli B., Lima J.M.N., Thomaz J. M. \& Carvalho A.F. 2011. [Teeth morphofunctional classification of coati, Nasua nasua.] Classificação morfofuncional dos dentes de quati, Nasua nasua. Pesquisa Veterinária Brasileira 31(5):447-451. Departamento de Ciências Morfológicas, Centro Universitário da Fundação de Ensino Octávio Bastos, Av. Doutor Octávio Bastos s/n, Jardim Nova São João, São João da Boa Vista, SP 13874-148, Brazil. E-mail: labvet01@unifeob.edu.br

Nasua nasua, also known as coati, is an omnivorous mammal with wide distribution in South America and found all over Brazil. The evolution adapted the teeth of the multiple mammals for most different diets. Through these variations it is possible to classify the vertebrates' teeth of vertebrates in classes, genera and families. The objective of this paper is to describe and classify morphologically the teeth of Nasua nasua, comparing them with the teeth of Canis familiaris described in the literature. Five adult male and female Nasua nasua were studied. Photographs of the oral cavity of two macerated skulls were taken for documentation of teeth position. From other three animals, teeth were processed for decalcification and embedded in paraffin for histology. It was possible to reveal macroscopically male and female differences in N. nasua, due to a sexual dimorphism. Female have smaller round canine teeth, and male canine teeth are larger than the female ones. The animals presented the dental formula $2 x(I 3 / 3, \mathrm{C} 1 / 1, \mathrm{P} 4 / 4, \mathrm{M} 2 / 2)$ (I=incisive, $\mathrm{C}=$ canine, $\mathrm{P}=$ premolar, $\mathrm{M}=$ molar), with a total of 40 teeth. Microscopically, the teeth were classified as brachyodont with enamel in the most external region, covering the dentine. This type of teeth does not erupt constantly as the hypsodonts, because the enamel stays intact until the time of eruption. Teeth were also classified as anelodont, with a limited period of growth characteristics.
\end{abstract}

INDEX TERMS: Dental formula, Nasua nasua, teeth, morphology, Procyonidae.

RESUMO.- O quati (Nasua nasua), é um mamífero onívoro, amplamente distribuído pela América do sul e encontrado em todo Brasil. A evolução provocou nos dentes dos mamíferos múltiplas variações, adequando às mais diversas dietas. Por essas variações nos dentes é possível classificar os vertebrados em ordem, gênero e família. Portanto este trabalho teve como objetivo descrever e classificar morfologicamente os dentes de machos e fêmeas de $N$. nausa, a fim de classificá-los e compará-los entre si e com a morfologia dos dentes de Canis familiaris descrita na literatura. Neste estudo foram utilizados cinco animais Nasua nasua, adultos, de ambos os sexos, cedidas pelo Criatório

\footnotetext{
${ }^{1}$ Recebido em 30 de julho de 2010.

Aceito para publicação em 4 de fevereiro de 2011.

2 Departamento de Ciências Morfológicas, Centro Universitário da Fundação de Ensino Octávio Bastos, Av. Doutor Octávio Bastos $\mathrm{s} / \mathrm{n}$, Jardim Nova São João, São João da Boa Vista, SP 13874-148, Brasil.

*Autor para correspondência: labvet01@unifeob.edu.br
}

Cientifico Cecrimpas (Unifeob). Dois deles macerados tiveram a cavidade oral exposta para a realização da documentação fotográfica da posição dos dentes, outros três, os dentes foram submetidos à descalcificação e processamento e embebição em parafina para observação histológica. $\mathrm{Na}$ cavidade oral de N. nasua, devido ao dimorfismo sexual, foi possível comparar a dentição do macho e da fêmea macroscopicamente. A fêmea apresentou dentes menores, caninos arredondados e o macho, caninos maiores. Os animais apresentaram a fórmula dentária $2 x(I 3 / 3, C 1 / 1, P 4 / 4$, $\mathrm{M} 2 / 2)$ (I=incisivos, $\mathrm{C}=$ caninos, $\mathrm{P}=$ pré-molares e $\mathrm{M}=$ molares) $=40$. Microscopicamente todos os dentes foram classificados como braquidontes, pois o esmalte é depositado sobre a dentina. Este tipo de dente não tem erupção constante como o hipsodonte, pois seu esmalte permanece intacto até a época de erupção. Seus dentes também foram classificados como anelodontes, por ter um período limitado de características de crescimento. 
TERMOS DE INDEXAÇÃO: Fórmula dentária, Nasua nasua, dentes, morfologia, Procyonidae.

\section{INTRODUÇÃO}

O quati (Nasua nausa) pertence ao filo Chordata, classe Mammalia, ordem Carnívora e à família Procyonidae, sendo um animal onívoro, de hábito diurno e encontrado amplamente na América do sul, ocupando quase todas as regiões do Brasil (Teixeira \& Ambrosio 2007). Estes animais por ser representantes da ordem carnívora, seus dentes são fortes e afiados que podem causar sérias lesões em outros animais (Teixeira \& Ambrosio 2007).

Os dentes são estruturas anatômicas calcificadas, adaptadas para o corte, a retenção e a trituração dos alimentos, tendo como função básica reduzir mecanicamente o tamanho dos alimentos (Madeira 2005). Na evolução dentária dos mamíferos, o conjunto de lentas alterações na arcada dentária permitiu a diferenciação desses animais em onívoros, herbívoros ou carnívoros e, em cada um desses grupos, surgiu uma ampla gama de formas dentárias (Cartelle 2008). Embora os dentes dos mamíferos sejam formados pelos mesmos componentes, esmalte, polpa e cemento, a dentição dos animais, em geral, apresentam-se variável de acordo com as diferentes espécies (Banks 1992).

Os mamíferos domésticos apresentam dentes classificados como heterodonte, pois apresentam diversos grupos de dentes, cada um possuidor de determinadas características, adaptadas a funções específicas (Junqueira \& Carneiro 2004).

Os incisivos são pequenos e situam-se bem unidos na parte rostal do maxilar e da mandíbula, estes dentes são usados para dividir o alimento (Dyce et al.1997).

Os dentes caninos, nos carnívoros, são usados principalmente para defesa de território e disputa de fêmeas, e são ausentes nos roedores (Sisson 1986, Dyce et al.1997).

Os pré-molares são dentes de perfuração ou de corte sendo destinados ao processamento do alimento (Sisson 1986). Os carnívoros ainda apresentam os dentes denominados "carniceiros", o último pré-molar superior e o primeiro molar inferior, os quais são usados principalmente para rasgar os alimentos (Freitas et al. 2008).

Os molares possuem um grande potencial cortante, porém são desenvolvidos principalmente para trituração. Alguns animais onívoros como Didelphis sp. apresentam o molar denominado tribosfênico, apresentando múltiplas faces e cúspides altamente pontiagudas, porém este tipo de dente promove um atrito dental muito acentuado, principalmente em animais jovem (Aguiar et al. 2004).

Os dentes podem ser classificados em duas formas braquidonte e hipsodonte, sendo o primeiro comumente observado em marsupiais como o gambá (Didelphis sp.) (Maçanares et al. 2004) e animais domésticos, nestes dentes o esmalte recobre toda a coroa (Banks 1992), já os hipsodontes são encontrados em roedores como o mocó (Kerodon rupestris) (Thomaz et al.2006) e em herbívoros, nestes o cemento recobre toda a coroa do dente (Banks 1992).
As estruturas mineralizadas do dente são: esmalte, dentina e cemento. $O$ esmalte é um tecido densamente calcificado e acelular, produzido pelos ameloblastos, células que morrem antes da erupção do dente (Gartner \& Hiatt 1997). A dentina é um material amarelado, produzida em forma de túbulos pelos odontoblastos, células que estão ao redor da polpa. Esta estrutura é sensível a diversos estímulos (Junqueira \& Carneiro 2004).

O cemento é uma estrutura menos rígida, semelhante ao osso imaturo, contendo os cementoblastos que são as células responsáveis pela produção de cemento durante toda a vida do dente (Gartner \& Hiatt 1997).

A parte central do dente é chamada de cavidade pulpar e seu formato se assemelha ao do próprio dente, sendo constituída de tecido conjuntivo frouxo, rico em proteína, com vários vasos, nervos, e apresentando alguns elementos circulatórios linfáticos. $\mathrm{Na}$ raiz pode ser observado $\mathrm{O}$ forame apical, uma abertura que permite a entrada de vasos e nervos na polpa (Gartner \& Hiatt 1997, Junqueira \& Carneiro 2004). O periodonto é a estrutura responsável pela fixação dos dentes no osso maxilar e mandibular. Os tecidos que o compõe são cemento, ligamento periodontal, osso alveolar e gengiva (Banks 1992, Junqueira \& Carneiro 2004).

Desta forma, observando a importância dos dentes na sobrevida dos animais silvestres e selvagens, este trabaIho objetivou analisar morfologicamente os dentes do macho e da fêmea de quati, através do estudo macro e microscópio com a finalidade de descrevê-los e classificálos morfologicamente.

\section{MATERIAL E MÉTODOS}

Para análise macro e microscópica foram utilizados cinco quatis adultos de ambos os sexos, fixados em formaldeído a 10\% em solução aquosa sendo que destes cinco, dois animais já macerados, todos provenientes do criatório científico Cecrimpas (Proc. IBAMA 02027.00286/04-92) - Centro de Criação, Multiplicação e Pesquisa em Animais Silvestres do Centro Universitário da Fundação de Ensino Octávio Bastos (Unifeob).

O procedimento executado para eutanásia em outros projetos encontra-se descrito a seguir, sendo que para este estudo os animais já se encontravam fixados, sem prejuízo para as técnicas empregadas.

Eutanásia: os animais foram eutanasiados, através a seguinte técnica: pré-anestesia com acepran $3 囚 0,05 \mathrm{mg} / \mathrm{kg}(\mathrm{IM})$, seguindo de indução anestésica com telasol4® 20mg/kg (IV). Após alcançar o plano anestésico profundo, foi injetada uma ampola de cloreto de potássio 19,1\% (10ml) via intracardíca.

Os animais foram monitorados através de estetoscópio e demais sinais vitais até a parada cardíaca completa. Imediatamente após a morte, os animais tiveram a aorta abdominal canulada e todo o sistema circulatório foi lavado com solução fisiológica de cloreto de sódio a 0,9\% e em seguida fixados com solução de formol a $10 \% \mathrm{pH} \mathrm{7,4.}$

Assim todos os animais eutanasiados, mediante aprovação de outras pesquisas perante apreciação do Comitê de Ética do Curso de Medicina Veterinária da Unifeob. Para a utilização dos animais fixados no Departamento de Anatomia Animal neste trabalho, novo pedido ao Comitê de Ética foi encaminhado e aprovado. 


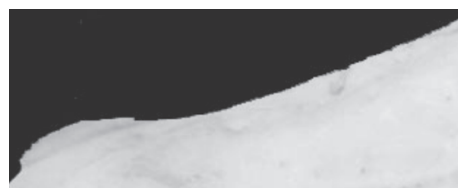

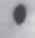

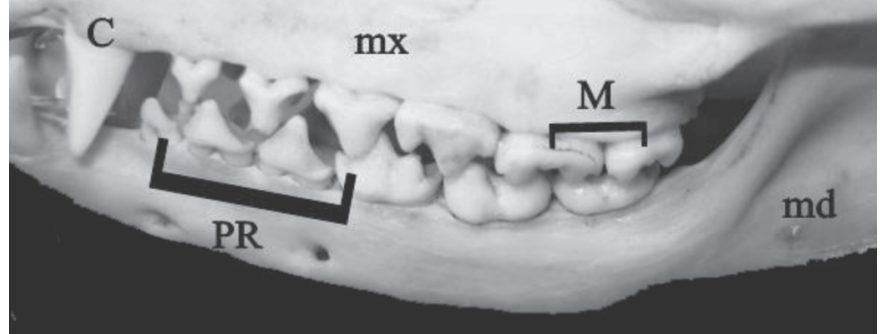

Fig.1. Vista lateral do crânio do quati, detalhe do maxilar (mx), mandíbula (md), dente canino superior $(\mathrm{C})$, pré-molares inferiores $(\mathrm{PR})$, molares superiores $(\mathrm{M})$.

Dos animais fixados no Departamento de Anatomia Animal, dois animais tiveram sua cavidade oral exposta com o auxílio de pinça anatômica, tesoura e bisturi, para que fossem realizadas a documentação fotográfica, edificação e mensuração com um paquímetro de precisão (Mitotoyo $®$ ).

Os dentes de quati foram retirados de dois exemplares (um macho e uma fêmea) previamente fixados em formol. Esse procedimento foi realizado com o uso de pinça anatômica, bisturi e tesoura. Desta forma, foram coletados oito dentes, sendo dois incisivos, dois pré-molares, dois molares e dois caninos, destes, quatro foram processados pela técnica de desgaste e observados ao microscópio óptico e outros quatro dentes foram submetidos à técnica de descalcificação modificada (Rodrigues 1998) descrita a seguir: $800 \mathrm{ml}$ de água destilada, $120 \mathrm{ml}$ de acido fórmico, $80 \mathrm{ml}$ de acido clorídrico.

Os dentes foram colocados em imersão na solução descalcificante a qual foi trocada a cada três dias. Diariamente, os dentes eram submetidos à pressão com agulha fina para verificar a capacidade de perfuração e conseqüente descalcificação.

No momento em que a agulha atravessava com facilidade a estrutura dentária (aprox. 20 dias) pode-se dar prossegui- mento ao processamento histológico rotineiro de embebição em parafina (Histosec $₫$ Merck), inclusão e corte em um micrótomo Leica RM2165®. Os corte foram feitos com espessura de 5 ìm e foram corados por hematoxilina-eosina (HE) (Tolosa et al. 2003).

Foram realizadas documentações fotográficas com auxílio de uma máquina Sony Mavica 3.2 Mp e a fotomicrográficas em um Fotomicroscópio Leica DM 2000.

\section{RESULTADOS}

$\mathrm{Na}$ cavidade oral, de todos os quatis analisados, foram encontrados 40 dentes, expressos pela fórmula dentária $2 x(13 / 3, \mathrm{C} 2 / 2, \mathrm{P} 4 / 4$ e $\mathrm{M} 2 / 2)=40$.

Os incisivos do quati são dentes simples, unirradiculares, unicuspidados e extremamente curtos. Porém os dois últimos incisivos superiores são mais longos que os rostrais. Os caninos foram classificados como os maiores dentes da boca, caracterizados por serem unirradiculares e, na sua oclusão, situaram-se uns ao lado do outro, mas não se apresentaram desgastados, além de serem dentes grande, curvos e lateralmente compridos, apresentando um formato trifacetado.

Os pré-molares são em número de três ou quatro em cada hemi-arcada, apresentando coroa longa e cúspides situadas centralmente e distalmente. São birradiculares ou trirradiculares. Macroscopicamente esses dentes apresentam tamanhos e complexidade crescente no sentido medio-distal (Fig.1).

Os molares dos animais neste estudo, são maiores que os pré-molares e apresentam varias cúspides, mas apenas duas são cortantes, conferindo um aspecto serrilhado quando observado lateralmente (Fig.1).

Comparando a dentição do macho e da fêmea de quati, macroscopicamente a fêmea apresentou dentes caninos menores (Fig.2A) e arredondados, enquanto no macho os caninos são maiores, pontiagudos com um formato trifacetado(Fig.2B). Esta diferença foi evidenciada pela mensuração dos dentes onde a média e o desvio padrão da fêmea são de $1 \mathrm{~cm}$ e $0 \mathrm{~cm}$ e no macho a média é o

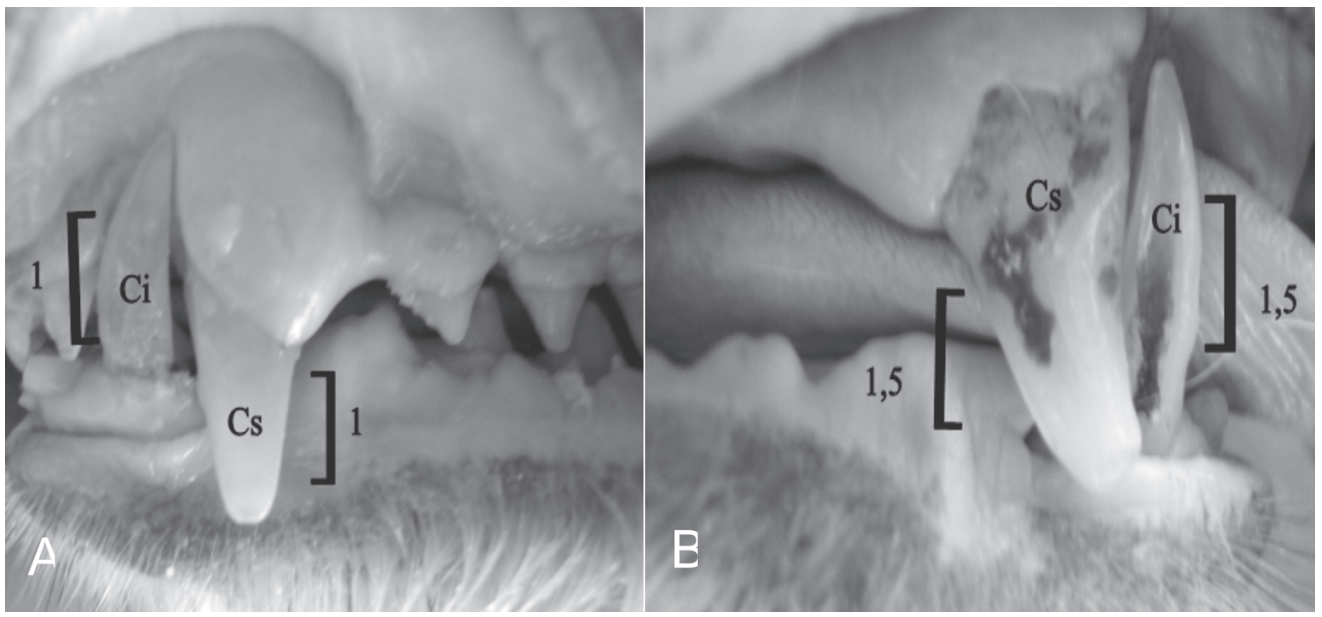

Fig. 2. (A) Vista lateral da cavidade oral do quati. Observar o tamanho do canino superior (cs) e do inferior (ci) da fêmea. (B) Canino superior (cs) e inferior (ci) do macho em centímetros. 


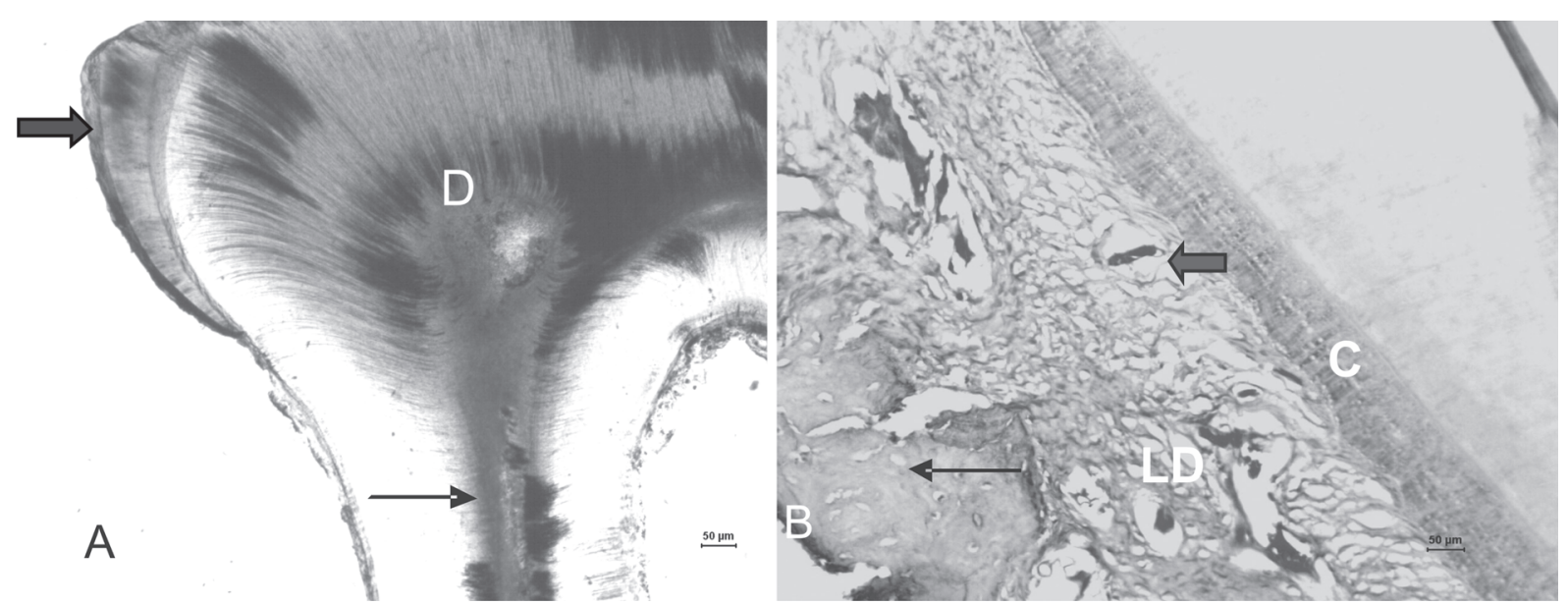

Fig.3. Os dentes de Nasua nasua. (A) O esmalte (seta cheia), a dentina e a polpa (seta fina), todos compondo o ápice do dente. Obj.2,5x. (B) As camadas que compõe o periodonto, osso alveolar (seta fina), o ligamento periodontal (LD) com vaso sanguíneo (seta cheia) e o cemento (C). Técnica de desgaste, descalcificação e coloração em HE, Obj.5x.

desvio pardrão são de $1,5 \mathrm{~cm}$ e $0 \mathrm{~cm}$, conforme mostra a Figura 2.

Todos os dentes do quati analisados neste estudo foram classificados como braquidontes, pois este apresentou a coroa recoberta por esmalte, e possuem bem definidas as regiões de raiz, coroa e colo.

Os animais estudados apresentaram dentes como esmalte, dentina, pré-dentina, polpa e periodonto (Fig.3A,B). Foi possível observar a dentina bem espessa com seus túbulos dentinários, que se tornam mais espessos e mais ramificados próximos ao esmalte.

$\mathrm{Na}$ raiz foi encontrado o forame apical e o cemento (Fig.3B) mais espesso na região apical. O periodonto foi observado em todos os dentes, sendo bem espesso nos dentes molares entre as duas raízes e constituído por ligamento periodontal (Fig.3B).

\section{DISCUSSÃO}

$\mathrm{Na}$ cavidade oral do quati foram encontrados 40 dentes, expessos pela fórmula dentária $2 \times(I 3 / 3, C 2 / 2, \mathrm{P} 4 / 4$ e M2/ 2) $=40$ conforme foi descrito por Teixeira \& Ambrosio (2007) nessa mesma espécie. Porém, de acordo com Freitas et al. (2008) é natural a variação no número de pré-molares nestes animais.

Estes autores afirmam que, em cativeiro, podem ser observadas doenças orais na cavidade oral que causam desgaste e fraturas, principalmente nos dentes caninos e incisivos e estes podem vir a modificar a sua fórmula dentária.

O quati, assim como o gambá (Didelphis sp.) (Aguiar 2004), é um animal onívoro e, portanto, seus dentes apresentam funções semelhantes. Os incisivos apenas prendem alimentos, os caninos perfuraram e os pré-molares e molares, trituraram e cortam os alimentos sem que haja contato oclusal entre eles.

Seus dentes incisivos foram classificados como dentes simples, com volume crescente no sentido medio-distal, por serem bem parecidos com os de cães, assim como descrito por Sisson (1986). Esta semelhança na dentição do cão e do quati refere-se principalmente por estes animais pertencerem à mesma ordem e possuírem os mesmos hábitos alimentares.

Os dentes caninos do quati são grandes, e por ser um animal da ordem carnívora, mas apresentar hábitos onívoros, utiliza estes dentes para fins agressivos, como defesa de território e disputa de fêmeas, hábito similar ao descrito por Sisson (1986), Dyce et al. (1997) nos cães, e Freitas et al. (2008) em quatis.

Os dentes molares e pré-molares são bem semelhantes, porém os molares são maiores e apresentam várias cúspides como o dos cães descrito por Sisson (1986).

No entanto, os dentes também foram classificados como bunodonte, que são os dentes a partir do quarto pré-molar caudalmente, tanto na mandíbula como no maxilar, esse tipo de dentes é encontrado em animais onívoros, como afirma Freitas et al. (2008) em seus estudos com esta espécie.

Os carnivoros apresentam os dentes denominados de sectorios ou carniceiros, estes no quati têm como principal função ragar o alimento, porém nos carnivoros doméstico tem como função moer os alimentos, assim estes animais apresentam carcteristicas semelhantes, porém com algumas funções diferentes, segundo os estudos de Dyce et al. (1997) em cães e Freitas et al. (2008) nesta espécie.

Comparando macroscopicamente a dentição do macho e da fêmea de quati macroscopicamente, a fêmea apresenta dentes menores em relação ao macho. Este fato pode estar relacionado aos estudos comportamentais feitos por Gompper (2004) e Russel (1996) os quais afirma que as fêmeas são matriarcas e vivem com seus filhotes em grupos de até 30 indivíduos, não utilizando os caninos para fins de defesa, mas apenas para a alimentação, enquanto que os machos, quando se tornam adultos, vivem isolados e só se aproximam do grupo na época do acasalamento, utilizando os caninos afiados para a disputa de fêmeas. 
Os dentes de Nasua nasua apresentam característica anelodonto, pois este tem um período limitado de característica de crescimento e como braquidontes, assim como os dentes dos marsupiais e dos animais domésticos, conforme afirmado por Banks (1992), Mançanares et al. (2004) e Freitas et al (2008), tendo em vista que, estes animais apresentam alimentação mais leve, promovendo menor desgaste aos dentes.

Todos os dentes de N. nasua analisados sob microscopia de luz são compostos por esmalte, dentina, pré-dentina, polpa e periodonto, conforme afirma Junqueira \& Carneiro (2004). O esmalte está presente na parte superior do dentes, assim como foi observado também no quati neste trabalho, sendo a estrutura mais rígida do corpo, porém é totalmente acelular, e incapaz de reagir a qualquer agressão, conforme afirma Banks (1992) e Gartner \& Hiatt (1997). A dentina foi observada em todo o dente, pois esta reveste a polpa dentária por isto ela é sensível a diversos estímulos, como afirmam Junqueira \& Carneiro (2004).

$\mathrm{Na}$ raiz dos dentes de quati, foi encontrado o forame apical, entrada de nervos e início da polpa; o cemento, estrutura semelhante ao osso, foi encontrado mais espesso na região apical, local no qual ocorre produção continua de cemento, para compensar o desgaste normal da coroa, devido o seu constante uso, conforme descrito por Banks em outras espécies (1992).

O periodonto do dente de quati é bem espesso e com muitos vasos, pois ele é responsável pela fixação do dentes no osso maxilar e mandibular, é composto pelo ligamento periodontal, formado por tecido conjuntivo denso, fibroblastos, fibras de colágeno e o osso alveolar imaturo, assim como descrito por Banks (1992), Junqueira \& Carneiro (2004) em outras espécies.

\section{CONCLUSÕES}

Macroscopicamente pode-se concluir que:

- A cavidade oral do quati é semelhante à do cão (Canis familiaris).

- A diferença entre machos e fêmeas de quatis, está no tamanho e formato dos dentes caninos inferiores e superiores.

- Os dentes de quati são braquidontes, apresentando a coroa recoberta pelo esmalte, além de apresentar coroa colo e raiz bem definidos. Todos os dentes são compostos por esmalte, dentina, pré-dentina, cavidade pulpar, polpa e periodonto.
Agradecimento.- Ao Centro Universitário da Fundação de Ensino Octávio Bastos pela disponibilização de todo o material para a realização deste estudo.

\section{REFERÊNCIAS}

Aguiar M.S., Ferigolo J., Junior J.L.R. \& Gioso M.A. 2004. Atrição dental em Didelphis albiventris e D. marsupialis (Marsupialia, Didelphimorphia, Didelphidae) do Sul do Brasil. Ciência Rural 42:11271132.

Banks W.J. 1992. Histologia Veterinária Aplicada. 2ª ed. Editora Manole, São Paulo. 560p.

Cartelle C. 2008. O dente. Ciência Hoje 41:28-33.

Dyce R.M., Sack W.O. \& Wensing C.J.G.1997. O Tratado de Anatomia Veterinária. $3^{\underline{a}}$ ed. Editora Guanabara Koogan, Rio de Janeiro, p.86-89.

Freitas E.P., Rahal S.C., Teixeira C.R., Teixeira R., Mendes G.M. \& Gioso M.A. 2008. Oral cavity evaluation and dental chart registration of coati (Nasua nasua) in captivity. J. Vet. Dentistry 25:110-117.

Gartner L.P. \& Hiatt J.L. 1997. Tratado de Histologia. Guanabara Koogan, Rio de Janeiro. 414p.

Gompper M.E. 1995. Nasua narica. Mammalian Species 487:1-10.

Junqueira L.C. \& Carneiro J. 2004. Histologia Básica. 9ª ed. Guanabara Koogan, Rio de Janeiro, p.415-431.

Madeira M.C. 2005. Anatomia do Dente. 4aㅡ ed. Editora Sarvier, São Paulo. 128p.

Mançanares C.A.F., Carvalho A.F., Casals J., Bellatine T., Thomaz J.M., Esteves A., Ambrósio C.E., Miglino M.A., Rosa R.A. \& Santos A.M.A.C. 2004. Análise macro e microscópica do dente do gambá, Didelphis sp. Reunião de Integração da Morfologia Pan-Americana, Foz do Iguaçu.

Rodrigues H. 1998. Técnicas Anatômicas. 2ª ed. Editora Arte Visual, Vitória. 222p.

Russel S.K. 1996. Teming of reproduction by coati (Nasua narica) in relation to fluctuations in food resourcis, p.413-431. In: Lugh E.G., Rand A.S. \& Windson D.M. (Eds), The Ecology of a Tropical Forest. $2^{\text {nd }}$ ed. Smithsonian Institution Press, Washington, USA.

Sisson S. 1986. Aparelho digestório, p.100-104. In: Getty R. (Ed.), Anatomia dos Animais Domésticos. $5^{\mathrm{a}}$ ed. Editora Guanabara Koogan, Rio de Janeiro.

Teixeira R.H.F. \& Ambrosio S.R. 2007. Carnívora, Procyonidae (mão pelada, quati, jupará), p.571-573. In: Cuba Z.S., Silva J.C.R. \& CatãoDias J.L. (Eds), Tratado de Animais Selvagens. Editora Roca, São Paulo.

Thomaz M.J., Carvalho A.F., Miglino M.A., Maçanares C.A.F., Ambrosio C.E. \& Moacir M.F. 2006. Caracterização morfológica dos dentes do mocó, Kerodon rupestris: Mammalia, Rodontia. Braz. J. Res. Anim. Sci. 46(4):702-707

Tolosa E.M.C., Rodrigues C.J., Behmer O. \& Freitas Neto A.G. 2003. Manual de Técnicas para Histologia Normal e Patológica. $2^{\mathrm{a}}$ ed. Manole, São Paulo, p.331. 